\title{
An Effective Torsion Model for Extra Gauge Bosons and Their Mass Corrections
}

\author{
J. A. Helayël-Neto*† \\ Centro Brasileiro de Pesquisas Físicas, \\ Rua Dr. Xavier Sigaud 150, Urca, \\ Rio de Janeiro, Brazil, CEP 22290-180 \\ E-mail: Gosehelavelegmail.com
}

\section{N. Panza}

Centro Federal de Educação Celso Suckow da Fonseca

Avenida Maracanã, 229, Maracanã

Rio de janeiro, Brazil, CEP 20271-110

E-mail:

\begin{abstract}
LHC provides an excellent laboratory to probe massive graviton effects in scenarios that may describe gravity at the $\mathrm{TeV}$ scale. Based on this fact, in the present work, we are interested in analyzing the possible constraints on the free parameters of the gravity Lagrangian in 4 dimensions recently studied in the work of Reference [U] by taking exclusively torsion effects into account, in order to generate mass for the graviton. We shall consider an effective field theory approach, in which the gravity is excited through its torsion modes, while metric effects are frozen in the regime of the quark-gluon plasma densities of the ALICE.
\end{abstract}

7th Conference Mathematical Methods in Physics - Londrina 2012,

16 to 20 April 2012

Rio de Janeiro, Brazil

* Speaker.

$\dagger$ A footnote may follow. 


\section{Introduction}

Beyond questions, Einstein's field theory accounts very well for almost all known macroscopic gravitational phenomena. However, as a quantum theory (ultra-violet scales) it is not a satisfactory description. The extensions of the General Relativity (GR), considering Lagrangians with powers of the curvature and torsion, did not prove to be sufficient to solve simultaneously the problems of renormalizability and unitarity of the theory. In this context, it is important to analyse the possible consequences of a propagating torsion in effective models. In spite of that, as shown in Ref. [四], even in this case, the interaction of the particles of the Standard Model (SM) with the propagating torsion leads to a violation of unitarity, after one-loop quantum corrections are included. Thus, for the effects of the dynamical torsion to be compatible with the phenomenology of the SM and with the idea of the so-called large extra dimensions (LEDs), we must assume that the characteristic mass scale of the torsion terms be much below the Plankian regime and the coupling between torsion and the SM fermions should not be neglected [ [2].

In Cosmology, the observed current accelerated expansion of the Universe that might be signaling a failure of GR at large distances (infra-red scales), is a compelling idea that motives the investigation of large-distance modifications of gravity. GR can be viewed as a theory of a massless spin-2 particles, gravitons, but does not excluded the question of whether gravity could be mediated by a small massive graviton. In this context, in Cosmology, the massive graviton dark matter scenario is considered as a possible alternative dark energy model to to account for the present accelerating universe [ [3], 团]. Furthermore, several peoples agree with the fact that unification between gravity and electromagnetism has to take into account torsion in four- and higherdimensional theories such as Kaluza-klein models. With all these arguments, it is unquestionable the importance of the torsion field in the description of gravity as a fundamental interaction. From the experimental point of view, the more exciting expectation is the production of a real massive graviton at LHC from hadronic process and the feasibility of detectation of quantum gravity effects at the Tev scale.

The aim of the present paper is to propose a unitary and free-from-tachyion model that generates mass for the graviton, assuming that the masse of graviton comes only from the excitation of the torsion degree of freedom, in the so-called Cartan restricted geometry. Specifically, we investigate, in this context, the model considered in Ref. [[]]. We work with the vielbein and the spin connection as independent fields. This choice is motivated because we think that it is a more fundamental approach to gravitation, since it is based on the fundamental ideas of the Yang-Mills approach.

This paper is organised as follows: in Section II we present a description of the model, our conventions and obtain the propagators of the corresponding models. Also, since that Dirac fields couple to the affine connection the intrinsic spin of fermions acts like a source of torsion. In Section III, we study an extension of our model in order to include the minimal coupling between torsion and Dirac spinors as well as between torsion and Majorana spinors. Section IV is devoted to our conclusive comments and new perspectives. 


\section{Description of the Model}

As it is well-known, in a space-time in which torsion is present, the metric and the affine connection can be, in general, considered independent fields. The last is non-symmetric and the torsion tensor is defined as $T_{\mu \nu}{ }^{\alpha}=\Gamma_{\mu \nu}{ }^{\alpha}-\Gamma_{\nu \mu}{ }^{\alpha}$. In four dimensions, the torsion tensor has 24 degrees of freedom and can be decomposed with respect to the Lorentz group into three irreducible tensors, namely: a pseudo-vector $a^{\lambda}$, a vector $v^{\mu}$ and a rank-three tensor $q_{\mu v \alpha}$, which satisfies the the conditions $q_{\mu \alpha}^{\alpha}=0$ and $\varepsilon^{\alpha \beta \mu v} q_{\alpha \beta \mu}=0$.

$$
T_{v \mu \alpha}=q_{v \mu \alpha}+\frac{1}{3}\left(\eta_{v \alpha} v_{\mu}-\eta_{\mu \alpha} v_{v}\right)+\varepsilon_{v \mu \alpha \lambda} a^{\lambda}
$$

Our purpose is to investigate the role of a propagating torsion in the description massive gravity with explic torsions terms. Within all possible quadratic terms that we can form with torsion, the independent contributions turn out to be $T_{\mu v}{ }^{a} T^{\mu v}{ }_{a}, T_{\mu a}{ }^{a} T^{\mu b}{ }_{b}, T_{a b c} T^{a b c}$. The most general parity-preserving Lagrangian without higher derivatives is given by [四:

$$
\begin{aligned}
\mathscr{L}_{g}= & e\left(-\frac{1}{\kappa^{2}} R+\chi R^{2}+\beta R_{\mu a} R^{\mu a}+\gamma R_{\mu a} R^{a \mu}+\xi R_{\mu v a b} R^{a b \mu v}+k R_{\mu v a b} R^{a b \mu v}\right. \\
& \left.+\lambda R_{\mu v a b} R^{\mu a v b}+s T_{\mu v}{ }^{a} T^{\mu v}{ }_{a}+t T_{\mu a}{ }^{a} T^{\mu b}{ }_{b}+r T_{a b c} T^{a b c}\right)
\end{aligned}
$$

where $e_{\mu}^{a}$ is the vielbein, $e=\operatorname{det} e_{\mu}^{a}$ and $R_{\mu \nu}{ }^{a b}$ is the field strength associated with the spin connection $\omega_{v}{ }^{a b}$. Moreover, $\chi, \beta, \gamma, \xi, k$ and $\lambda$ are arbitrary dimensionless constants and the others parameters have canonical dimensions given by $[s]=[t]=[r]=1$. Our conventions are [[5]:

$$
\begin{gathered}
R_{\mu \nu}{ }^{a b}=\partial_{\mu} \omega_{\nu}{ }^{a b}-\partial_{\nu} \omega_{\mu}{ }^{a b}+\omega_{\mu}{ }^{a}{ }_{c} \omega_{\nu}{ }^{c b}-\omega_{\nu}{ }_{c}{ }_{c} \omega_{\mu}{ }^{c b} \\
R_{\mu}{ }^{a}=e_{b}^{v} R_{\mu \nu}{ }^{a b} \\
R=e_{a}^{\mu} e_{b}^{v} R_{\mu \nu}{ }^{a b} \\
\eta_{a b}=(1,-1,-1,-1)
\end{gathered}
$$

where the Geek indices refer to the world manifold and the Latin ones stand for the frame indices. Before we proceed, we recall that the spin connection $\omega_{a b c}$ may be expressed as

$$
\omega_{\mu}^{a b}=\widetilde{\omega}_{\mu}^{a b}+K_{\mu}^{a b}
$$

which $\widetilde{\omega}_{\mu}{ }^{a b}$ is the Riemannian part of the spin connection

$$
\widetilde{\omega}_{\mu}^{a b}=\frac{1}{2} e_{\mu c}\left(\Omega^{c a b}+\Omega^{a c b}-\Omega^{b a c}\right)
$$

where $\Omega_{c b a}=e_{c}^{\mu} e_{b}^{v}\left(\partial_{\mu} e_{v a}-\partial_{v} e_{\mu a}\right)$ stands for the rotation coefficients. $K_{\mu}{ }^{a b}$ are the components of the contorsion tensor

$$
K_{\mu}^{a b}=\frac{1}{2}\left(T_{\mu}^{a b}-T^{a b}{ }_{\mu}+T^{b}{ }^{a}\right)
$$

As we consider only quadratic terms in the curvature and torsion in the Lagrangian, by virtue of the Gauss-Bonet theorem, 


$$
\int d^{4} x e\left(R_{\mu v a b} R^{\mu v a b}-4 R_{\mu a} R^{\mu a}+R^{2}\right)=0
$$

there is a redundant term among the possibilities for $\mathrm{D}=4$.

Adopting the fact that we are working on a energy scale in which the gravity is excited by the torsion, but not by the metric, the vielbein can be expressed as a Kronecker delta, so that $\widetilde{\omega}_{\mu}{ }^{a b}=0$.

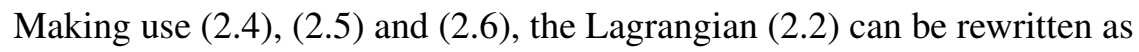

$$
\begin{aligned}
\mathscr{L}_{g} & =-\alpha R+\beta R_{\mu v} R^{\mu v}+\gamma R_{\mu v} R^{v \mu}+\xi R_{\mu v \alpha \beta} R^{\mu v \alpha \beta}+k R_{\mu v \alpha \beta} R^{\alpha \beta \mu \nu} \\
& \left.+\lambda R_{\mu v \alpha \beta} R^{\alpha \beta \mu v}+s T_{\mu \alpha \beta} T^{\mu \alpha \beta}+t T_{\mu \alpha \beta} T^{\alpha \beta \mu}+r T_{\mu \alpha}{ }^{\mu \beta} T_{\beta}\right)
\end{aligned}
$$

Throughout the paper, we shall consider the Cartan restricted geometry, in which $q_{v \mu \alpha}=0$. The physical meaning of this assumtion is that the tensor component of the torsion is not coupled to the Dirac spinor field, as discussed in Ref. [[]]. Concerning the spin connection $\omega_{\mu}{ }^{a b}$, one gets the explicit expression

$$
\omega_{\beta \mu \nu}=\frac{1}{3}\left(\eta_{\beta v} v_{\mu}-\eta_{\mu \beta} v_{v}\right)+\frac{1}{2} \varepsilon_{\beta \mu \nu \lambda} a^{\lambda}
$$

In order to investigate the spectrum of our model, we split the bilinear piece of the Lagrangian as:

$$
\mathscr{L}_{g}=\frac{1}{2}\left(v^{\mu} a^{\mu}\right) O_{\mu v}\left(\begin{array}{c}
v^{v} \\
a^{v}
\end{array}\right)
$$

where the Hermitian bilinear form $O_{\mu \nu}$ has the form

$$
O_{\mu v}=\left(\begin{array}{cc}
(b \square+f) \theta_{\mu v}+((b+c) \square+f) \omega_{\mu v} & 0 \\
0 & (e \square+g) \theta_{\mu v}+((e+d) \square+g) \omega_{\mu v}
\end{array}\right)
$$

$\theta_{\mu v}=\eta_{\mu v}-\frac{\partial_{\mu} \partial_{v}}{\square}$ being the projector on the transverse vector states and $\omega_{\mu v}$ the projector on the longitudinal vector states. The elements of the matrix operator ([D. 1 (I) are given by:

$$
\begin{gathered}
b=-\frac{4}{9}(2 \beta+4 \xi+\lambda) \\
c=-\frac{8}{3}\left(\frac{2 \beta}{3}+\gamma+\frac{\xi}{3}+k+\frac{\lambda}{3}\right) \\
d=\gamma+2 \xi+4 k-3 \lambda-\beta \\
e=-\gamma+4 \xi-4 k+\beta \\
f=\frac{2}{3}(2 \alpha+2 s-t+3 r) \\
g=-3(\alpha+4 s+4 t)
\end{gathered}
$$

The non-zero propagators in momentum space are listed below,

$$
i\langle v v\rangle=\frac{9 /[4(2 \beta+4 \xi+\lambda)]}{p^{2}-m_{1}^{2}} \theta_{\mu v}+\frac{3 /[4(2 \beta+2 \xi+\lambda+2 \gamma+2 k)]}{p^{2}-m_{2}^{2}} \omega_{\mu v}
$$


where

$$
\begin{gathered}
m_{1}^{2}=\frac{3(t-2 s-3 r-2 \alpha)}{2(2 \beta+4 \xi+\lambda)}, m_{2}^{2}=\frac{t-2 s-3 r-2 \alpha}{2(2 \beta+2 \xi+\lambda+2 \gamma+2 k)} \\
i\langle a a\rangle=\frac{1 /(\gamma-4 \xi+4 k-\beta)}{p^{2}-m_{3}^{2}} \theta_{\mu v}+\frac{1 /[3(\lambda-2 \xi)]}{p^{2}-m_{4}^{2}} \omega_{\mu v}
\end{gathered}
$$

where

$$
m_{3}^{2}=\frac{3(4 s+4 t+\alpha)}{(\gamma-4 \xi+4 k-\beta)}, m_{4}^{2}=\frac{4 s+4 t+\alpha}{\lambda-2 \xi}
$$

Now that we have obtained the spectrum of our model, we must search for the possible choise of the parameters in the Lagrangian (2.8), such that the massive gravitons belong to the Tev scale. As in the next section will be interested only in the pseudo-vector component of the torsion, in the sequel we present the condition that must be satisfied in order to get a unitary model with a propagating massive graviton by analysing eq. (15).

$$
s=-\frac{\alpha}{4}, \gamma-4 \xi+4 k-\beta>0, t>0, \quad \lambda-2 \xi>0
$$

This correspond to the following Lagrangian:

$$
\begin{aligned}
\mathscr{L}_{g} & =-\alpha R+\beta R_{\mu v} R^{\mu v}+\gamma R_{\mu v} R^{v \mu}+\xi R_{\mu v \alpha \beta} R^{\mu v \alpha \beta}+k R_{\mu v \alpha \beta} R^{\alpha \beta \mu \nu} \\
& +\lambda R_{\mu v \alpha \beta} R^{\alpha \beta \mu v}-\frac{\alpha}{4} T_{\mu \alpha \beta} T^{\mu \alpha \beta}+t T_{\mu \alpha \beta} T^{\alpha \beta \mu}+r T_{\mu \alpha}{ }^{\alpha} T^{\mu \beta}{ }_{\beta}
\end{aligned}
$$

where the masses of propagating modes are:

$$
m_{3}^{2}=\frac{12 t}{\gamma-4 \xi+4 k-\beta}, \quad m_{4}^{2}=\frac{4 t}{\lambda-2 \xi}
$$

\section{Coupling With Fermionic Matter Fields}

In recent years, the study of gravity with torsion in presence of fermionic massive matter has received a great deal of attention in connection with effective low-energy limit of strings which yield dynamics for the spin connection.. Futhermore, as it is also well-known from Einstein-CartanSciama-Kibble (ECSK) gravity, the torsion becomes a dynamical variable related to the spin density of matter [2]. In this section, we shall be concerned with the minimal coupling between fermions and torsion, assuming that the metric fluctuations are taken to be very week as compared to the torsion excitations, in the model discussed in section I. We tackle the issue a 1-loop mass generation mechanism for the fermion, considered as a Majorana particle. In this case, the interaction between the fermion and the torsion takes place by the pseudo-vector component $a_{\mu}$.

The requeriment that the Dirac equation in a gravitational field preserve local Lorentz invariance yields a direct interaction between torsion and fermions, so that in a Riemann-Cartan space-time, the Dirac Lagrangian for a massive fermion has the form

$$
\mathscr{L}_{D}=\frac{i}{2} e e_{a}^{\mu} \bar{\psi} \gamma^{a} D_{\mu} \psi-\frac{i}{2} e e_{a}^{\mu}\left(D_{\mu} \bar{\psi}\right) \gamma^{a} \psi-e m_{0} \bar{\psi} \psi
$$

where the covariant derivative of the spinor field are given by

$$
D_{\mu}=\partial_{\mu}+\frac{i g}{8} \omega_{\mu}^{a b}\left[\gamma_{a}, \gamma_{b}\right]
$$


and $g$ is a dimensionless coupling constant, $\gamma^{a}=\gamma^{\mu} e_{\mu}^{a}$ with $\gamma^{\mu}$ denoting Dirac matrices and $m_{0}$ is the mass of the Dirac field.

In order to rewrite the Dirac Lagrangian in terms of the vector and pseudo-vector components of the torsion, we make use of the identity

$$
\gamma^{a} \gamma^{b} \gamma^{c}=\gamma^{a} \eta^{b c}+\gamma^{c} \eta^{a b}-\gamma^{b} \eta^{a c}+i \varepsilon^{a b c d} \gamma_{5} \gamma_{d}
$$

for obtain, after some algebra

$$
\mathscr{L}_{D}=i \bar{\psi} \gamma^{\mu} \partial_{\mu} \psi-m_{0} \bar{\psi} \psi+\frac{g}{2} \bar{\psi} v^{\alpha} \gamma_{\alpha} \psi+\frac{3 i g}{4} \bar{\psi} \gamma_{5} a_{\lambda} \gamma^{\lambda} \psi
$$

Now, we can extract the Feynman rules for the theory. The propagator for the pseudo-vector is given by eq.

$$
i \Delta_{0}(p)=\frac{A}{p^{2}-m_{4}^{2}} \eta_{\mu v}+\left(\frac{B}{p^{2}-m_{3}^{2}}-\frac{A}{p^{2}-m_{4}^{2}}\right) \frac{p_{\mu} p_{v}}{p^{2}}
$$

where $A=1 /(\gamma-4 \xi+4 k-\beta)>0$ and $B=1 /[3(\lambda-2 \xi)]>0$, and for the spinor field is given by

$$
i S_{0}(p)=\frac{1}{p^{\prime}-m_{0}}
$$

Futhermore, the fermion-torsion vertex is given by

$$
V_{\lambda}=i \frac{3 g}{4} \gamma_{5} \gamma_{\lambda}
$$

From these results, we can calculate the self-energy corrections for the bare propagators and discuss the mechanism of mass generation for fermion fields. For the fermion self-energy graph, we find:

$$
\begin{gathered}
-i \Sigma(p)=(i)^{4}\left(\frac{3 g}{4}\right)^{2} \int \frac{d^{4} q}{(2 \pi)^{4}} \gamma_{5} \gamma^{\mu} \frac{p^{\prime}-q+m_{0}}{(p-q)^{2}-m_{0}^{2}} \gamma_{5} \gamma^{v}\left[-\frac{A}{p^{2}-m_{4}^{2}} \eta_{\mu v}+\right. \\
\left.\left(\frac{A}{p^{2}-m_{4}^{2}}-\frac{B}{p^{2}-m_{3}^{2}}\right) \frac{q_{\mu} q_{v}}{q^{2}}\right]
\end{gathered}
$$

By a direct calculation, we obtain

$$
\Sigma(p)=\frac{9 A m_{0}^{2}}{32 \pi^{2} m_{4}^{2}} \ln \Lambda^{2} g^{2} \mu^{-\frac{1}{2 \ln \Lambda^{2}}}\left(\not p-m_{0}\right)+\text { finite }
$$

where $\mu$ is a arbitrary mass parameter and $\Lambda$ is a cutoff. The finite part of the correction to the propagator is not very important in the following discussion, and therefore is omitted.

Making use of the unrenormalized proper self-energy part of equation (B.9), $\widetilde{\Sigma}(p)$, we can express the observed mass $m$ in terms of the bare mass $m_{0}$, coupling constant $g$ and cutoff $\Lambda$.

$$
m-m_{0}=\left.\widetilde{\Sigma}(p)\right|_{\not \prime=m}
$$

or explicitly 


$$
m-m_{0}=\left(m-m_{0}\right) \frac{9 A m_{0}^{2}}{32 \pi^{2} m_{4}^{2}} g_{\text {eff }}^{2}
$$

where $g_{\text {eff }}=g\left(\frac{\ln \Lambda^{2}}{\mu^{1 /\left(2 \ln \Lambda^{2}\right)}}\right)^{1 / 2}$ is a effective coupling constant.

Our explicit calculations indicate that the gravitational coupling of the fermion to the pseudovector associated to torsion degrees of freedom is not able to dynamically induce mass. We check that, by setting $m_{0}=0, m$ turns out to vanish. So dynamical mass generation does not take place. so, the only possible effect of the coupling to $a^{\mu}$ is a mass shift upon radiative corrections.

We then adopt the following scenario. The tree-level mass, $m_{0}$, of the fermion must have been generated by means of the spontaneous breakdown of some (local) internal symmetry through a higgs field. We suppose some extra massive higgs of the MSSM [Q] acquired a vacuum expectation value and endowed the fermion with mass $m_{0} \sim 1 \mathrm{Tev}$. Then, by invoking naturalness [ㅁ] ], we expect a shift of, at most, the same order. This means that the gravitational coupling shoud produce a shift of the order of $1 \mathrm{Tev}$. With that physical assumption, our 1-loop computations yield the constraint below for the effective gravitational coupling, $g_{\text {eff }}$ :

$$
g_{\text {eff }} \leq \frac{4 \pi}{3} \sqrt{\frac{2}{A}}
$$

However, here a non-trivial fact occurs: the shifted mass, $m$, happens to dropout of our gap-like equation. Therefore, the most we can infer is a bound on $g$ set up by $m_{0}^{2}$. it is very unexpected that, at the very end, only $m_{0}^{2}$ remains in the would-be gap equation. The coupling to thje vector boson, a gauge-type interaction, is the responsible for this result. Actually, it is not generally proved, but only 4-fermion interactions give rise to dynamical mass generation.

\section{Concluding Comments}

We have taken in our approach the viewpoint that torsion is the only carrier of the gravity degrres of freedom. Fluctuations of the vielbein could also be taken into account that yield mixing terms between the emerging effective vector bosons [ $[$ ]. This would allow us to build up complex combinations of real gauge bosons to include extra charged mediators in our proposal. For that, the parameters should be such that they allow a degeneracy in mass between the charged gauge vectors that are the anti-particle of one another. We shall be reporting on this development in a forthcoming paper.

On the other hand, we expect, on the basis of our considerations of the phenomenology of quark-gluon plasmas at ALICE/LHC and RHIC, that the effects of the mixings shoud be rather small with respect to the contributions that torsion alone yields. This is so because the average energy density is very high [ए]] and fluctuations of the vielbein/metric become less relevant, for we expect the density of gravitons to be high. But, anyway, we believe, as already anticipated, that, even if the torsion fluctuations should dominate over the metric excitation modes, we should exploit in more details the mixings, to understand how the torsion fluctuations could eventually strengthen the metric fluctuations. We keep in mind to go further and to pursue an investigation on 
the vielbein-spin conection mixings in terms of the vector bosons that may be produced as effective fluctuations and to undestand possible effects of the mixings at the Tev scale.

\section{Acknowledments}

The authors express their gratitude to Ilya Shapiro, Sam Friot and David Greynat for a critical reading of the original manuscript.

\section{References}

[1] C. A. Hernaski, A. A. Vargas-Paredes, J. A. Helayël-Neto, Phys. Rev. D80: 124012, 2009.

[2] G. de Berredo-Peixoto, J.A. Helayel-Neto, I.L. Shapiro, JHEP 0002 (2000) 003 ; A. S. Belyaev, I. L. Shapiro and M. A. B. do Vale, Phys.Rev. D75 (2007) 034014; I.L. Shapiro, Phys. Repts., 357 (2002) 113.

[3] S. Capozziello, R. Cianci, C. Stornaiolo, Class. Quant. Grav. 24:6417-6430, 2007.

[4] Hyung Won Lee, Kyoung Yee Kim, Yun Soo Myung, General Relativity and Quantum Cosmology (gr-qc); Cosmology and Extragalactic Astrophysics (astro-ph. CO), 2001.

[5] V. de Sabbata and M. Gasperini, Introduction to Gravitation (World Scientific, Singapore,1985).

[6] Eric A. Bergshoeff, J.J. Fernández-Melgarejo, Jan Rosseel, Paul K. Townsend, JHEP (2012).

[7] J. L. Boldo, J. A. Helayel-Neto, N. Panza, Class.Quantum Grav. 19 (2002) 2201 .

[8] Nikodem J. Poplawski, Gen.Relativ.Gravit.44:491-499, 2012

[9] R. Foot and X.-G. He, Phys. Lett. B267 (1991) 509; F. Del Aguila, G. D. Coughlam and M. Quiros, Nucl. Phys. B307, (1988) 633; B. Holdom, Phys. Lett. B259 (1991) 329; M.Gasperini, Phys. Lett. B263 (1991) 267.

[10] E. Scapparone, "Latest results from ALICE", arXiv:1111.2685 [nucl-ex], on behalf of the ALICE Collaboration.

[11] Nikodem J. Poplawski, Phys. Rev. D 85, 107502 (2012). 